\title{
Immunotherapy in relapsed mesothelioma
}

\author{
Solenn Brosseau ${ }^{1,2}$, Xavier Dhalluin ${ }^{4,5}$, Gerard Zalcman ${ }^{1,2,3}$ \& Arnaud Scherpereel ${ }^{\star}, 4,5$ \\ ${ }^{1}$ Thoracic Oncology Department \& Early Trial Clinical Research Center, Hospital Bichat-Claude Bernard, Assistance \\ Publique-Hôpitaux de Paris (AP-HP), F-75018 Paris, France \\ ${ }^{2}$ University Paris-Diderot, Paris, France \\ ${ }^{3}$ U830 Inserm, 'Genetics \& Biology of Cancers', Research Center-Institut Curie \\ ${ }^{4}$ Pulmonary \& Thoracic Oncology, Univ Lille, CHU Lille, INSERM U1019, CIIL, Institut Pasteur de Lille, F59000 Lille, France \\ ${ }^{5}$ French National Network of Clinical Expert Centers for Malignant Pleural Mesothelioma Management (MESOCLIN), Lille, France \\ * Author for correspondence: arnaud.scherpereel@chru-lille.fr
}

'There is a strong rationale for immunotherapy in patients with MPM, in particular immune checkpoint inhibitors (ICI). The inflammatory phenotype of this tumor hints at the involvement of tumor-infiltrating T cells, while MPM cells express PD-L1 in a substantial proportion of cases”

First draft submitted: 11 October 2017; Accepted for publication: 26 October 2017; Published online: 20 December 2017

Malignant pleural mesothelioma (MPM) is a rare but aggressive loco-regional cancer arising from the parietal pleura, mainly after occupational asbestos exposure. MPM prognosis remains poor with median OS ranging from 13 to 19 months, in the 2004 pemetrexed registration trial [1], or the 2016 MAPS Phase III when combining anti-VEGF antibodies (bevacizumab) with standard first-line chemotherapy by cisplatin/pemetrexed [2], respectively. Thus, this triple combination may provide a new standard first-line treatment in patients fitted for bevacizumab. Conversely, no second-line strategy has been approved to date, except rechallenging the patients with long-lasting tumor control after first-line treatment with pemetrexed-based chemotherapy [3,4]. In fact, gemcitabine or vinorelbine are often used in this setting, but they exhibited very limited activity [5]. Moreover, almost all experimental therapies tested as second or further line treatment failed to show any substantial activity, with disease control rates (DCR) at 3 months of treatment lower than 30\%, and median overall survival (mOS) ranging from 6 to 9 months [3].

There is a strong rationale for immunotherapy in patients with MPM, in particular immune checkpoint inhibitors (ICI). The inflammatory phenotype of this tumor hints at the involvement of tumor-infiltrating $\mathrm{T}$ cells, while MPM cells express PD-L1 in a substantial proportion of cases [6-9]. Finally, PD-L1 tumor expression is correlated with worse prognosis in MPM patients [10,11].

The Phase I trial KEYNOTE-028 investigating anti-PD-1 antibody (Ab) pembrolizumab had yielded promising outcome in 25 patients, with a DCR of 72\%, a 5.5-month median progression-free survival (mPFS), and an mOS amounted to 18 months [12]. The Chicago Phase II trial with pembrolizumab had subsequently showed an interim analysis of the first 34 patients accrued (out of 65 planned), with a 6.2 months median PFS, DCR of $80 \%$ and mOS of 11.9 months [13]. The NivoMes study yielded very similar encouraging results in 34 patients treated by anti-PD-1 Nivolumab with a 50\% 3-month DCR and a 3.6-month mPFS [14]. Finally, the anti-PD-L1 Avelumab (Javelin trial) also exhibited activity in MPM patients (any line of treatment) with DCR of 57\% [15]. In contrast, in the multicenter, randomized, double-blind, placebo-controlled Phase IIB DETERMINE trial ( $\mathrm{n}=571$ patients), the anti-CTLA-4 tremelimumab did not significantly improve second- and third-line MPM patients' outcome compared with placebo, yielding a 7.7 months (95\%CI [6.8-8.9]) mOS versus 7.3 months (95\%CI [5.9-8.7]), respectively $(\mathrm{HR}=0.92$ [95\% CI: 0.76-1.12]; $\mathrm{p}=0.41)$ [16].

Therefore, the French Collaborative Thoracic Intergroup (IFCT) launched a randomized, noncomparative, Phase II MAPS-2/IFCT1501 trial evaluating nivolumab (N) $3 \mathrm{mg} / \mathrm{kg} / 2$ weeks $(\mathrm{n}=63)$ and the combination of nivolumab and anti-CTLA-4 Ab ipilimumab $(\mathrm{N}+\mathrm{I}) 1 \mathrm{mg} / \mathrm{kg} / 6$ weeks $(\mathrm{n}=62)$, until disease progression, unacceptable toxicity or maximum 2 years. The patients with unresectable MPM and documented progression after one or two previous lines of chemotherapy including pemetrexed/platinum doublet were enrolled. In each arm, PD-L1 expression status was available in 79\% of patients. The primary end point was the DCR at 12 weeks, targeting DCR $\geq 40 \%$ in each arm on the first 108 eligible patients, as centrally assessed by an independent panel. This trial was positive for both arms, with meaningful increases of DCR at 12 weeks in both $\mathrm{N}+\mathrm{I}$ arm

Future Medicine 
(50.0\%) and $\mathrm{N}$ arm (44.4\%) [17] compared with historical data and previous nonimmunotherapy clinical trials. Updated findings from the MAPS-2 trial were presented at the ESMO 2017 Congress [18]. According to a pooled analysis of patients with available PD-L1 status, centrally assessed PD-L1 expression $\geq 1 \%$ significantly correlated with objective response, and high PD-L1 expression ( $\geq 25 \%)$ correlated with both objective response and disease control. Median duration of response was 7.9 and 7.4 months in the N + I and N arms, respectively. Long-lasting remissions were observed for all mesothelioma histological subtypes. After a median follow-up of 15 months, median OS was 13.6 months $95 \% \mathrm{CI}$ (6.7-Not reached) in the monotherapy arm, and not even reached in the combination arm. Mature mPFS results for $\mathrm{N}+\mathrm{I}$ and $\mathrm{N}$ alone arms were 5.6 months $95 \% \mathrm{CI}[3.2-8.4]$ and 4.0 months $95 \%$ CI [2.8-5.7], respectively, after the extended follow-up showing the maturity of such analyses. Toxicity of both regimens was generally manageable. In the combo arm, there were three deaths deemed treatment-related, due to fulminant hepatitis, encephalitis and acute kidney failure, occurring early in the study course, with no more grade 5 event thereafter, suggesting the investigators improved their caring for immune-related AEs during the study proceeding. For the majority of documented immune-related AEs, nonsignificantly higher rates were noted in the $\mathrm{N}+\mathrm{I}$ arm, but most of these were grades 1 and 2.

Thus, results of MAPS-2 support the recent NCCN panel decision to recommend nivolumab monotherapy or the nivolumab plus Ipilimumab combination as options for second- or third-line treatment in relapsing MPM patients. The US FDA also just granted the orphan drug status to nivolumab, and to nivolumab plus ipilimumab combination, in this setting.

Based on early trials' results, pembrolizumab started to be used as off-label treatment for relapsed MPM in Switzerland. A Swiss registry assessed the real-life value of pembrolizumab in these patients in 13 cancer centers [19]. Most patients had pembrolizumab at a flat dose of $200 \mathrm{mg}$ every 3 weeks. Among 48 registered patients, one and 11 achieved complete or partial responses, respectively, leading to ORR of $25 \%$. With 13 patients achieving SD, DCR was $52 \%$. Median PFS and OS in this cohort were 3.6 and 7.2 months, respectively. $67 \%$ of 37 patients having available tissue samples for of these were PD-L1 expression considered as negative $(<5 \%)$, while 22 and $11 \%$ of these patients had PD-L1 tumor expression of $5-49 \%$ and $\geq 50 \%$, respectively. The PD-L1-positive subgroups showed four- to five-fold higher ORRs compared with the PD-L1-negative cohort. The patients with PD-L1 expression $\geq 50 \%$ achieved a DCR of $100 \%$. Likewise, PFS and OS improved with increasing PD-L1 expression. Seven patients (15\%) discontinued pembrolizumab treatment due to AEs.

The NIBIT Meso 1 trial assessed the value of anti-PD-L1 Durvalumab combined with Tremelimumab in patients as first $(30 \%)$ or second $(70 \%)$ line treatment [20]. Main end point, immune related ORR, was $27.5 \%$; mOS was 15.3 months, consistent with MAPS-2 and other trials [20].

Taken all together, the results of these studies accumulating more than 250 patients obviously showed significant activity of ICI, with acceptable toxicity. Thus, one could reasonably question whether the next step should be a placebo-controlled Phase III trial. However, a double-blinded, randomized, Phase III trial comparing nivolumab monotherapy versus placebo has been launched in second-line MPM patients, sponsored by the Southampton University (UK), with OS as primary end point (NCT03063450). It should ultimately confirm the efficacy of nivolumab in such setting, provided the recruitment plan of 336 patients would not be impaired by the spectacular results of MAPS2 trial, which could actually raise some ethical issues on the placebo arm. Alternatively, as previously observed for rare cancer diseases, one could defend a temporary authorization for ICI use, with prospective survey of efficacy and toxicity. A definitive authorization, could then come from the results of the ongoing company-sponsored randomized Phase III trial (Checkmate 743, NCT02890299) comparing first-line combination ICI therapy to pemetrexed-platinum doublet, which should provide enough safety data in 600 patients, as definitively assess the efficacy of ICI in MPM patients. Other clinical research design could also be favored such as anti-PD-1 association with pemetrexed-based chemotherapy, similarly as the ongoing 'Dream' trial in Australia (Cis/Pem/Durvalumab).

There are presently other immunotherapies than ICI evaluated in MPM. However, to date, strategies targeting mesothelin did not show significant efficacy. Vaccines and cell or gene therapies are still under investigation after promising preliminary results. Finally, anti-PD-1 or anti-PD-L1 Ab are now evaluated in Phase I trials in combination with a antiangiogenic drugs (bevacizumab or nintedanib), or with epigenetic agents (defactinib), or even with antimesothelin immune-conjugate (Anetumab ravtansine).

Although conflicting results arose from PD-L1 biomarker studies, there is currently no ideal biomarker to enable us to define the subset of patients benefiting at best from ICI. As in other cancers, the current search of other potential predictive biomarker for immunotherapy in MPM include assessments of tumor infiltration by immune 
cells, tumor mutational burden and neo-antigens, whole genome sequencing and gut microbiota evaluation. Finding such biomarkers is a crucial issue to counterbalance the risk of (immune) side effects and the costs of immunotherapy. Thus, malignant pleural mesothelioma research is actually at a crossroad, the breakthrough of immunotherapies and targeted therapies being close to deeply modify therapeutic strategies of this cruel disease.

\section{Financial \& competing interest disclosure}

S Brosseau has no relevant affiliation of financial involvement with any organization or entity with a financial interest in or financial conflict with the subject or materials discussed in the manuscript. This includes employment, consultancies, honoraria, stock ownership or options, expert testimony or patents received or pending, or royalties. X Dhalluin was invited by Astra-Zeneca at the 2017 ASCO meeting in Chicago. X Dhalluin, G Zalcman and A Scherpereel are investigators of Checkmate 743 Phase III trial sponsored by BMS. BMS purchased Nivolumab and Ipilimumab in MAPS-2 trial and French Intergroup IFCT perceived a research subvention from BMS for biomarker analyses in MAPS2 trial. G Zalcman perceived reimbursements for AACR Philadelphia meeting 2015 attendance and accommodation from BMS, and the 'Fondation AP-HP pour la Recherche' perceived a financial compensation for G Zalcman's participation to a BMS and MSD advisory boards. A Scherpereel has been also investigator in Phases I, II and III clinical trials in malignant pleural mesothelioma sponsored by Astra-Zeneca/Medlmmune, Bayer, Boehringer-Ingelheim, Epizyme and Verastem, with no personal payment, all honoraria being perceived by my Institution (CHU de Lille, Clinical Research Center, France). He was invited at some recent oncology international meetings (AACR, ASCO, WCLC) by Roche, MSD and BMS. He got honoraria for participation to some scientific or advisory Boards, organized by Astra-Zeneca, BMS, Boehringer-Ingelheim, MSD and Roche. The authors have no other relevant affiliations or financial involvement with any organization or entity with a financial interest in or financial conflict with the subject matter or materials discussed in the manuscript apart from those disclosed.

No writing assistance was utilized in the production of this manuscript.

\section{References}

1 Vogelzang NJ, Rusthoven JJ, Symanowski J et al. Phase III study of pemetrexed in combination with cisplatin versus cisplatin alone in patients with malignant pleural mesothelioma. J. Clin. Oncol. 21, 2636-2644 (2003).

2 Zalcman G, Mazieres J, Margery J et al. Bevacizumab for newly diagnosed pleural mesothelioma in the Mesothelioma Avastin Cisplatin Pemetrexed Study (MAPS): a randomised, controlled, open-label, Phase III trial. Lancet 387, 1405-1414 (2016).

3 Scherpereel A, Astoul P, Baas P et al. Guidelines of the European Respiratory Society and the European Society of Thoracic Surgeons for the management of malignant pleural mesothelioma. Eur. Respir. J. 1; 35(3), 479-495 (2010).

4 Baas P, Fenell D, Kerr KM, Van Schill PE, Haas RL, Peters S. Malignant pleural mesothelioma: ESMO clinical practice guidelines for diagnosis, treatment and follow-up. Ann. Oncol. 26(Suppl. 5), v31-v39 (2015).

5 Zauderer MG, Kass SL, Woo K, Sima CS, Ginsberg MS, Krug LM. Vinorelbine and gemcitabine as second- or third-line therapy for malignant pleural mesothelioma. Lung Cancer 84(3), 271-274 (2014).

6 Thapa B, Salcedo A, Lin X et al. The immune microenvironment, genome-wide copy number aberrations, and survival in mesothelioma. J. Thorac. Onco. 12(5), 850-859 (2017).

7 Lantuejoul S, Le Stang N, Damiola F, Scherpereel A, Galateau-Sallé F. PD-L1 testing for immune checkpoint inhibitors in mesothelioma: for want of anything better? J. Thorac. Oncol. 12(5), 778-778 (2017).

8 Mansfield AS, Roden AC, Peikert T et al. B7-H1 expression in malignant pleural mesothelioma is associated with sarcomatoid histology and poor prognosis. J. Thorac. Oncol. 9(7), 1036-1040 (2014).

9 Khanna S, Thomas A, Abate-Daga D et al. Malignant mesothelioma effusions are infiltrated by CD3+ T cells highly expressing PD-L1 and the PD-L1+ tumor cells within these effusions are susceptible to ADCC by the anti-PD-L1 antibody avelumab. J. Thorac. Oncol. 11(11), 1993-2005 (2016).

10 Cedrés S, Ponce-Aix S, Zugazagoitia J et al. Analysis of expression of programmed cell death 1 ligand 1 (PD-L1) in malignant pleural mesothelioma (MPM). PLoS One 10(3), e0121071 (2015).

11 Combaz-Lair C, Galateau-Sallé F, McLeer-Florin A et al. Immune biomarkers PD-1/PD-L1 and TLR3 in malignant pleural mesotheliomas. Hum. Pathol. 52, 9-18 (2016).

12 Alley E, Lopez J, Santoro A et al. Clinical safety and activity of pembrolizumab in patients with malignant pleural mesothelioma (KEYNOTE-028): preliminary results from a non-randomised, open-label, phase 1b trial. Lancet Oncol. 18, 623-630 (2017).

13 Kindler H, Karrison T, Tan YHC et al. Phase II trial of pembrolizumab in patients with malignant mesothelioma (MM): interim analysis. WCLC abstract OA13.02 (2016).

14 Quispel-Janssen J, Zimmermann M, Buikhuisen S, Burgers G, Baas P. A phase II study of nivolumab in malignant pleural mesothelioma NivoMes): with translational research (TR) biopsies. WCLC, abstract OA13.01 (2016).

15 Hassan R, Thomas A, Patel M et al. Avelumab (MSB0010718C; anti-PD-L1) in patients with advanced unresectable mesothelioma from the JAVELIN solid tumor phase Ib trial: Safety, clinical activity, and PD-L1 expression. J. Clin. Oncol. ASCO; abstract 8503 (2016). 
16 Maio M, Scherpereel A, Calabrò L et al. Tremelimumab as second-line or third-line treatment in relapsed malignant mesothelioma (DETERMINE): a multicenter, international, randomised, double-blind, placebo-controlled phase 2b trial. Lancet Oncol. 18, 1261-73 (2017).

17 Scherpereel A, Mazières J, Greiller L et al. Second or third-line nivolumab versus nivolumab plus ipilimumab in malignant pleural mesothelioma patients: results of the IFCT-1501 MAPS-2 randomized phase 3 trial. ASCO, abstract LBA8507 (2017).

18 Zalcman G, Mazieres J, Greiller L et al. Second or 3rd line nivolumab (nivo) or nivo plus ipilimumab in malignant pleural mesothelioma (MPM) patients: up-dated results of the IFCT-1501 MAPS1 randomized phase 2 trial. ESMO abstract LBA58_PR (2017).

19 Mauti LA, Klingbiel D, Schmid S et al. Pembrolizumab as second or further line treatment in relapsed malignant pleural mesothelioma; a Swiss registry. ESMO abstract 1615 O (2017).

20 Calabro L, Morra A, Giannarelli D et al. Tremelimumab plus Durvalumab in first-or secondline mesothelioma patients: final analysis of the NIBIT-MESO-1 Study. WCLC, abstract 9202 (2017). 